\title{
Supporting Information for: Force Based Canonical Approximation of Molecular Potentials: Average Force versus Pointwise Force
}

\author{
Jay R. Walton ${ }^{1}$ \\ Department of Mathematics, Texas A\&M University, College Station, TX 77843-3368, USA \\ Luis A. Rivera-Rivera ${ }^{2}$ \\ Department of Physical Sciences, Ferris State University, Big Rapids, MI 49307, USA
}

This Supporting Information covers details of the expansion of eq. (16) using three-term, five-term, and seven-term approximations for $\mathrm{H}_{2}, \mathrm{CH}^{+}, \mathrm{OH}$ and $\mathrm{He}$ dimer (only a six-term expansion is used for He dimer). Figures S1-S4 below exhibit the rate at which this process converges to tight approximations. The separation distances used are given in Tables 3-6.

\footnotetext{
${ }^{1}$ E-mail: jwalton@math.tamu.edu

${ }^{2}$ E-mail: LuisRiveraRivera@ferris.edu
} 


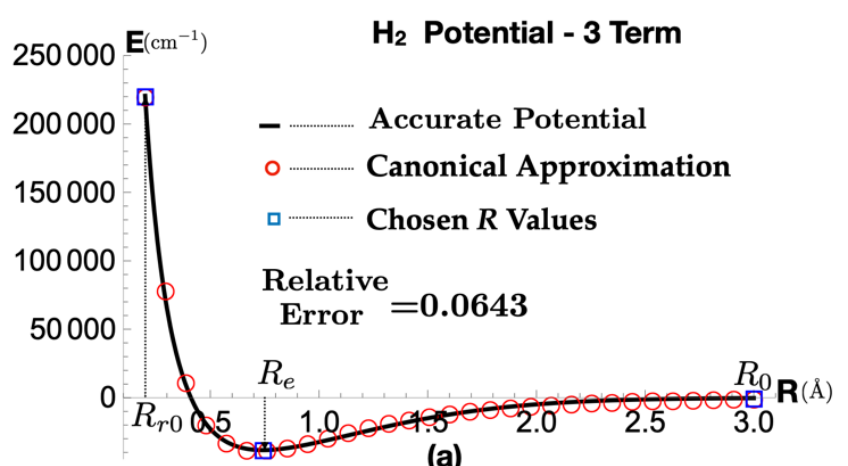

(a)
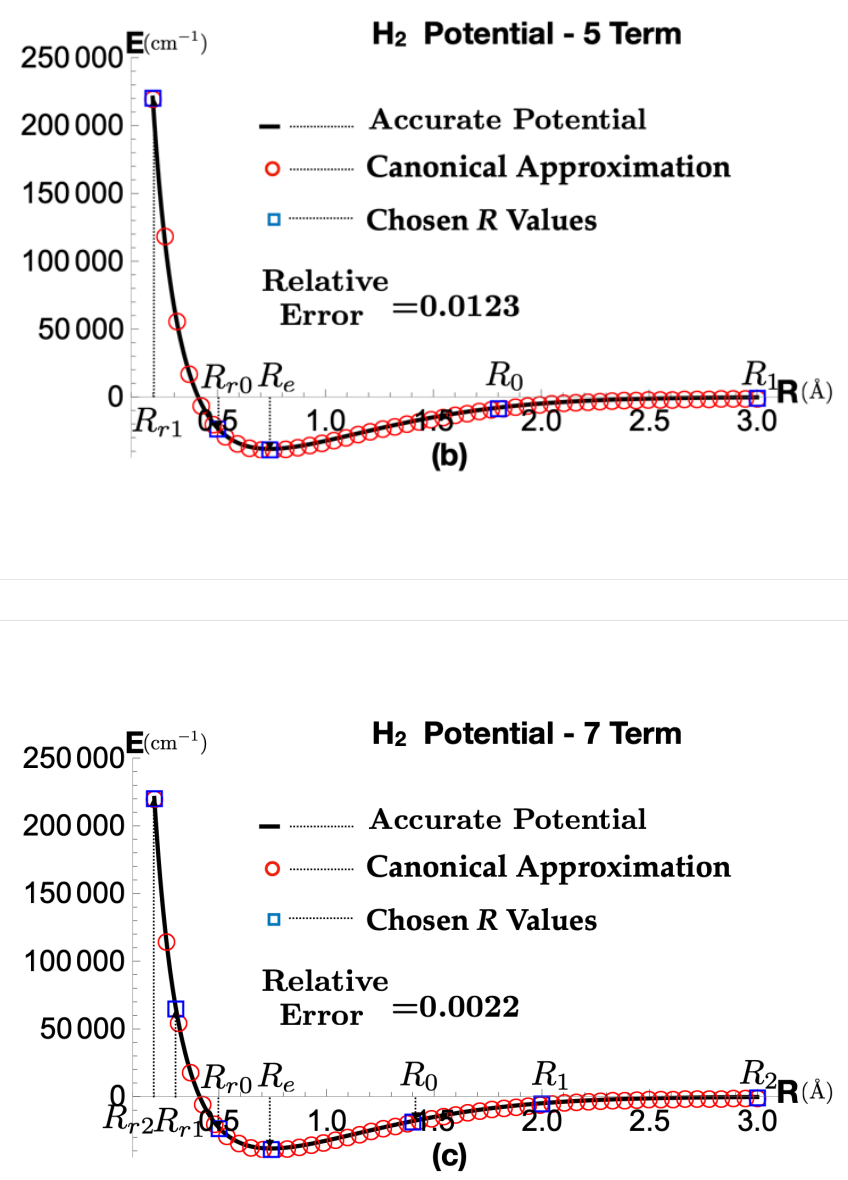

Figure S1. Three-, five-, and seven-term approximations for the $\mathrm{H}_{2}$ potential using the separation distances in Table 3 and eq. (16). 

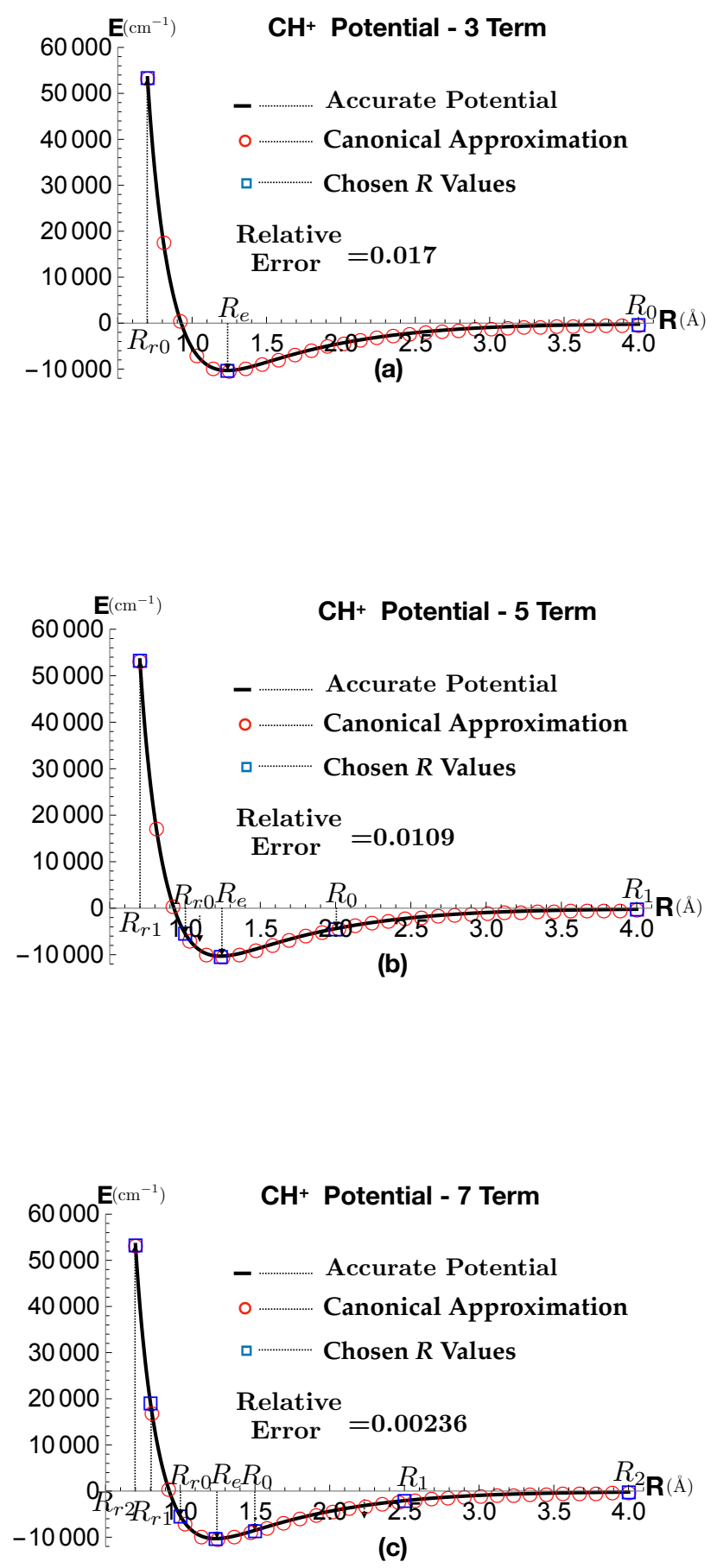

Figure S2. Three-, five-, and seven-term approximations for the $\mathrm{CH}^{+}$potential using the separation distances in Table 4 and eq. (16). 

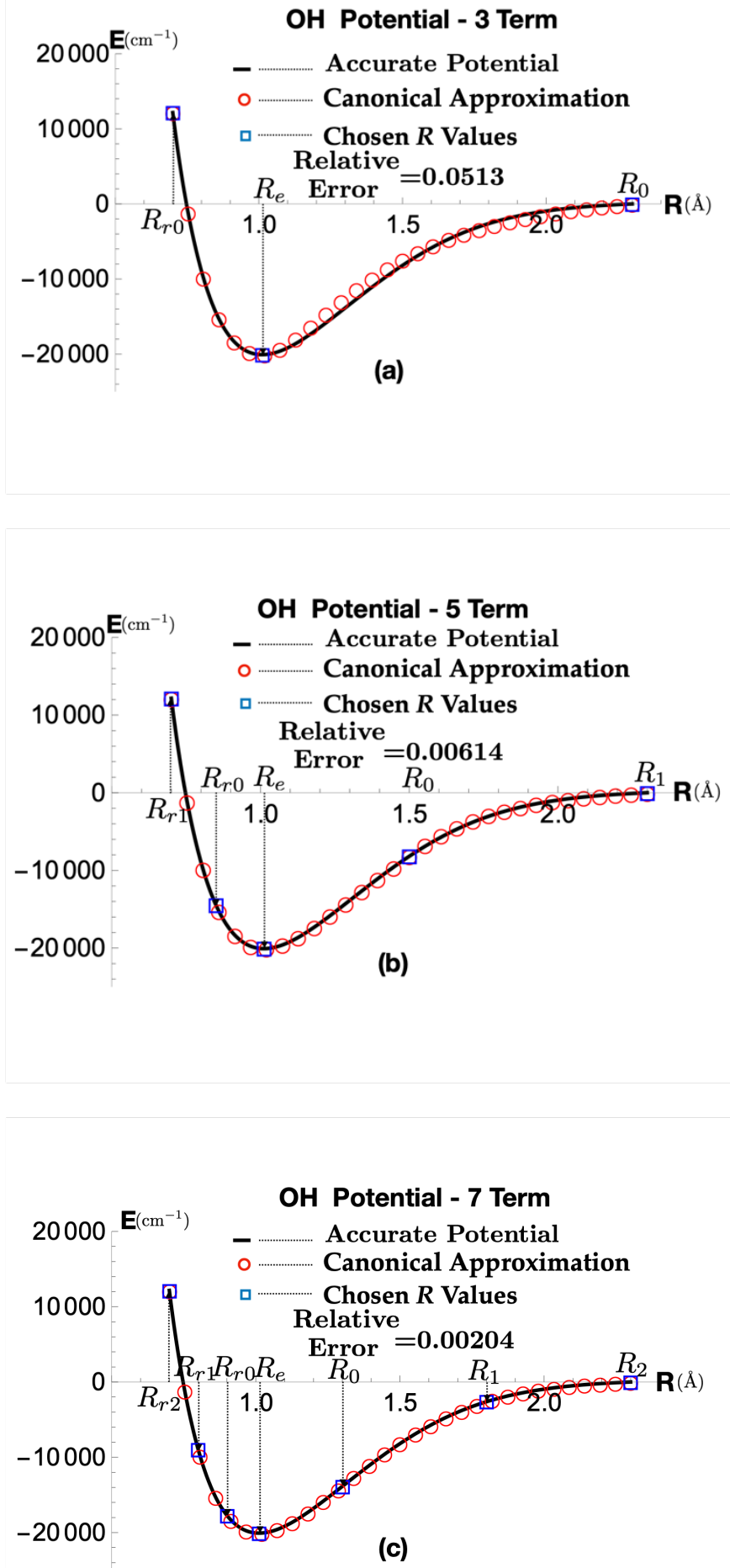

Figure S3. Three-, five-, and seven-term approximations for the $\mathrm{OH}$ potential using the separation distances in Table 5 and eq. (16). 

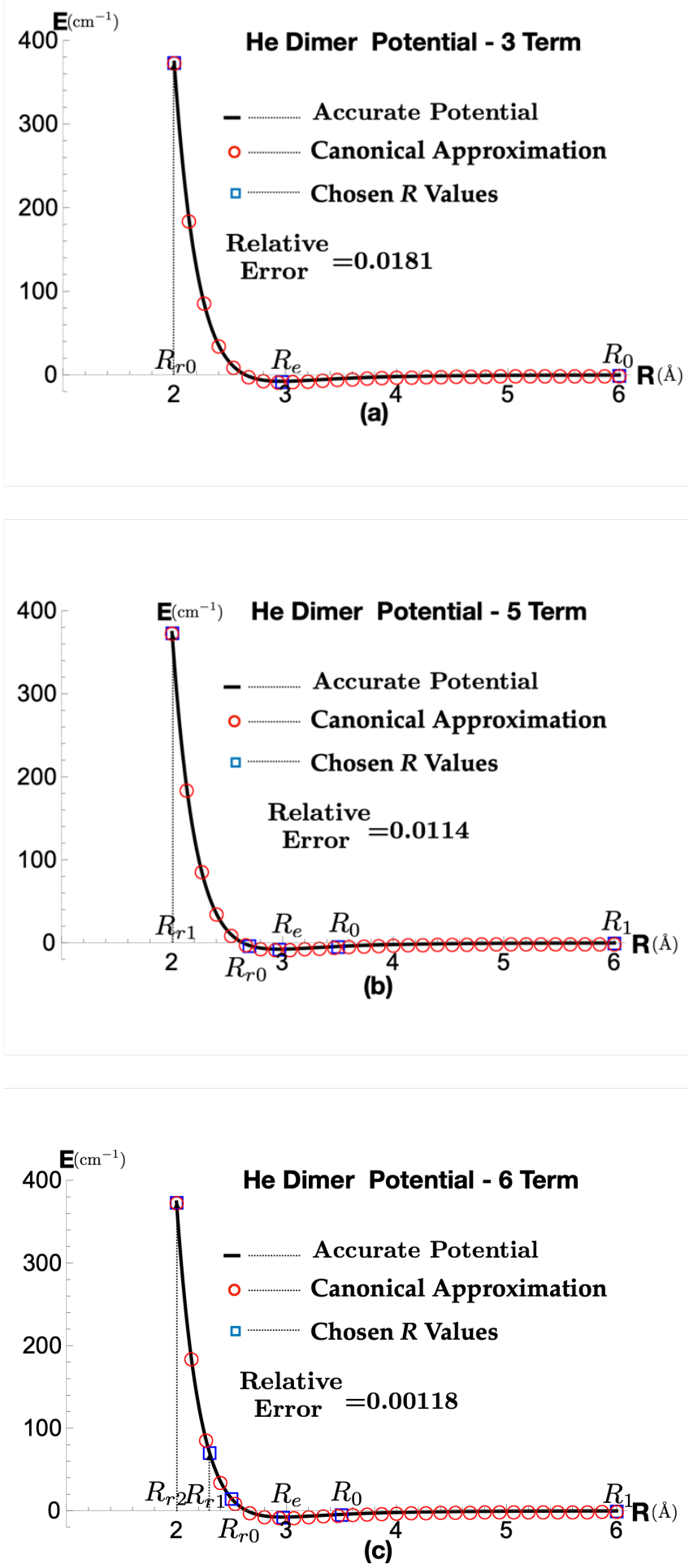

Figure S4. Three-, five-, and six-term approximations for the Helium dimer potential using the separation distances in Table 6 and eq. (16). 